\title{
The role of biological therapy in metastatic colorectal cancer after first-line treatment: a meta-analysis of randomised trials
}

\author{
E Segelov ${ }^{1}$, D Chan ${ }^{\star 2}$, J Shapiro ${ }^{3}$, T J Price ${ }^{4}$, C S Karapetis ${ }^{5}$, N C Tebbutt ${ }^{6}$ and N Pavlakis ${ }^{2}$ \\ ${ }^{1}$ St Vincent's Clinical School, University of New South Wales, Sydney, NSW 2052, Australia; ${ }^{2}$ Royal North Shore Hospital, St \\ Leonards, Sydney, NSW 2065, Australia; ${ }^{3}$ Monash University and Cabrini Hospital, Melbourne, VIC 3800, Australia; ${ }^{4}$ The Queen \\ Elizabeth Hospital and University of Adelaide, Woodville South, SA 5011, Australia; ${ }^{5}$ Flinders University and Flinders Medical \\ Centre, Flinders Centre for Innovation in Cancer, Bedford Park, SA, 5042, Australia and ${ }^{6}$ Austin Health, VIC 3084, Australia
}

Purpose: Biologic agents have achieved variable results in relapsed metastatic colorectal cancer (mCRC). Systematic meta-analysis was undertaken to determine the efficacy of biological therapy.

Methods: Major databases were searched for randomised studies of mCRC after first-line treatment comparing (1) standard treatment plus biologic agent with standard treatment or (2) standard treatment with biologic agent with the same treatment with different biologic agent(s). Data were extracted on study design, participants, interventions and outcomes. Study quality was assessed using the MERGE criteria. Comparable data were pooled for meta-analysis.

Results: Twenty eligible studies with 8225 patients were identified. The use of any biologic therapy improved overall survival with hazard ratio (HR) 0.87 (95\% confidence interval $(\mathrm{Cl}) 0.82-0.91, P<0.00001)$, progression-free survival (PFS) with $\mathrm{HR} 0.71(95 \% \mathrm{Cl}$ $0.67-0.74, P<0.0001)$ and overall response rate (ORR) with odds ratio (OR) $2.38(95 \% \mathrm{Cl} 2.03-2.78, P<0.00001)$. Grade $3 / 4$ toxicity was increased with OR 2.34. Considering by subgroups, EGFR inhibitors (EGFR-I) in the second-line setting and anti-angiogenic therapies (both in second-line and third-line and beyond settings) all improved overall survival, PFS and ORR. EGFR-I in third-line settings improved PFS and ORR but not OS.

Conclusions: The use of biologic agents in $\mathrm{mCRC}$ after first-line treatment is associated with improved outcomes but increased toxicity.

The efficacy of biologic agents or 'molecular targeted therapy' in first-line treatment of metastatic colorectal cancer (mCRC) has been extensively investigated. Their role in second-line therapy and beyond, however, has not been systematically examined. For this review, biological agents were defined as drugs targeting specific cancer cell growth factors, receptors and molecular pathways including the epidermal growth factor receptor (EGFR) and angiogenesis pathways.

The objective of this systematic review and meta-analysis was to identify, describe and summarise the benefits of biologic therapies in addition to standard care in patients with mCRC following progression during or after first-line systemic therapy. Two clinical scenarios were addressed: biologic therapy added to standard treatment compared with standard treatment alone (chemotherapy in second line, best supportive care (BSC) in third line and beyond); and biologic therapy added to standard treatment compared with a different biologic therapy added to same standard. The systematic review examined the effect of biologic agents on overall survival (OS), progression-free survival (PFS), and overall response rate (ORR) and documented toxicity and quality of life (QOL).

\section{*Correspondence: Dr D Chan; E-mail: dlhchan1@gmail.com}

Received 18 March 2014; revised 7 June 2014; accepted 16 June 2014; published online 29 July 2014 


\section{MATERIALS AND METHODS}

Search strategy. The authors agreed on study protocol prior to literature search and analysis although this was not centrally registered. The search strategy comprised analysis of: MEDLINE 1946-May 2012, EMBASE 1974-April 2012, BIOSIS 1992-May 2012 and COCHRANE reviews until May 2012 (for details, see Supplementary Methods) to identify relevant published randomised Phase II and III studies.

Proceedings of the American Society of Clinical Oncology Annual Scientific Meeting 2009-2013, ASCO Gastrointestinal Cancers Symposium 2010-2013 and the European Society for Medical Oncology Annual Scientific Meeting 2009-2012 were searched by hand. For studies available in abstract only, investigators were contacted for required information.

Patient characteristics. Studies involved patients with histologically confirmed mCRC who had received at least one prior line of chemotherapy for advanced disease. The trials investigated the addition of biological agent to chemotherapy, compared with either chemotherapy alone (Group 1) or the addition of a second biological agent to the same chemotherapy (Group 2).

Study review and inclusion. Two authors (ES/NP) independently reviewed titles and abstracts and agreed on articles to be retrieved. Studies included were registered RCTs evaluating second- or thirdline (or beyond) therapy for $\mathrm{mCRC}$, which reported at least one of the following: OS, PFS, ORR and toxicity.

Given the demonstrated efficacy of EGFR inhibitors (EGFR-I) to KRAS wild-type (WT) patients, only analysis of this population within EGFR-I trials was included. Potential studies were assessed independently by two reviewers (JS/ES) blinded to authors, journal, sponsor and results. Disagreement was resolved by a third reviewer (NP). Bias was assessed using the MERGE criteria. (Liddle et al, 1996) Two reviewers (NP/ES) independently extracted the most recent data from identified trials.

In order to perform a systematic review, categorisation of similar trials was required. Agents were categorised according to mode of action into the following subgroups:

- The monoclonal antibody EGFR-I: Cetuximab (Cunningham et al, 2004; Karapetis et al, 2008) and panitumumab, (Amado et al, 2008; Peeters et al, 2010; Hecht et al, 2012; Seymour et al, 2013). Anti-angiogenesis agents directed against vascular endothelial growth factor (VEGF): The monoclonal antibody bevacizumab (Giantonio et al, 2007; Hecht et al, 2012), the VEGF-trap agent aflibercept (Van Cutsem et al, 2012), and the VEGF receptor tyrosine kinase inhibitors (VEGFR TKIs) regorafenib (Grothey et al, 2013), brivanib (Siu et al, 2013) and vatalanib (PTK/ZK) (Van Cutsem et al, 2011).

- Newer targeted agents with mixed or novel mechanisms of action (target): Conatumumab (Death Receptor 5), ganitumumab and dalotuzumab (IGF-1 R), rilotumumab (HGF), tivantinib (MET), sorafenib (VEGFR/PDGFR/RAF) and vandetanib (VEGF/EGF/RET).

For the clinical scenario of the addition of one biologic agent compared with another, only three trials were identified, investigating these agents and targets: axitinib (VEGF 1/2/3), cediranib (VEGFR TKI), and panitumumab.

Outcome measures and data analysis. The outcomes of OS, PFS, ORR, toxicity and QoL were analysed based on trial-level data. Details of prior therapy could not be extracted from trial publications. Given possible data pointing toward differing efficacy by line of chemotherapy, analysis was stratified into second-line trials and trials investigating third-line (or later) setting. For the newer targeted agents, given most data were in predominantly second-line or mixed settings, a decision was made to analyse results as a single group. Results were reported according to PRISMA guidelines.

Data analysis was performed using REVMAN 5 for Windows. Individual RRs were pooled and $95 \%$ confidence interval (CI) was generated using the Mantel-Haenszel fixed-effects method. Unfortunately, analysis by prior therapy was not possible given usage of trial-level rather than individual patient data.

Meta-analysis of the log hazard ratio (HR) and log upper and lower CIs was performed. The outcome was considered statistically significant if $95 \%$ CI for relative risk did not cross 1 .

OS and PFS. For each trial, a HR and corresponding standard error was calculated. This was computed by the software in all cases except for Yang et al (2009) where PFS was derived by hand from the $80 \% \mathrm{CI}$.

$O R R$. This was calculated as the proportion of patients who achieved partial or complete response. Odds ratios (OR) for response were generated and the individual ratios pooled to give a clinically useful measure of effect.

Toxicity. Data were extracted on incidence of Grade 3 and 4 toxicity combined and Grade 5 toxicity separately with OR and pooled difference in toxicity calculated as for ORR. Detailed statistical analysis for risk of toxicity is presented for the combined cohort. Subgroup analyses are presented in Supplementary data.

Where there were $>2$ arms in a study, the study was entered twice in the data set (i.e. treated as two separate trials) with the number in the control group divided such that the total number added up to the original group size (as recommended by Cochrane Collaboration; The Cochrane Collaboration).

Heterogeneity was assessed using $\chi^{2}$ and $I^{2}$ tests, with values of $P<0.10$ and $I^{2}>50 \%$ indicating substantial heterogeneity. Where this was identified, the reasons were explored and DerSimonianLaird random-effects model performed for that outcome. This accounts imperfectly for heterogeneity, and must be taken with caution when the number of trials is less than five (Kontopantelis et al, 2013). Sensitivity analyses and funnel plots were undertaken to investigate possible bias.

Quality of life. Where available, QoL data were abstracted and the instrument noted. As there was insufficient data for quantitative analysis, QOL end points were reported descriptively.

\section{RESULTS}

Study selection. The literature search identified 218 citations; conference abstracts yielded an additional 331 references. Thirtyfour papers representing 20 studies comprising 8225 patients (Table 1, Supplementary Figure 1) were eligible for inclusion in the meta-analysis. Three pivotal studies were excluded: BOND (Cunningham et al, 2004), as cetuximab was administered in both arms; BOND2, as cetuximab and bevacizumab were administered in both arms; and EPIC, as analysis by KRAS status was available for only 300/1298 patients, with incomplete OS and PFS data (HRs only without CIs).

Risk of bias. The overall quality of the studies was good. Funnel plots (Supplementary Figures) show relative symmetry, arguing against significant publication bias, for all parameters except ORR. Here, the imbalance is not considered biologically plausible (i.e. significant worsening of ORR with addition of biologic), hence likely represents chance. 
Table 1. Study Characteristics

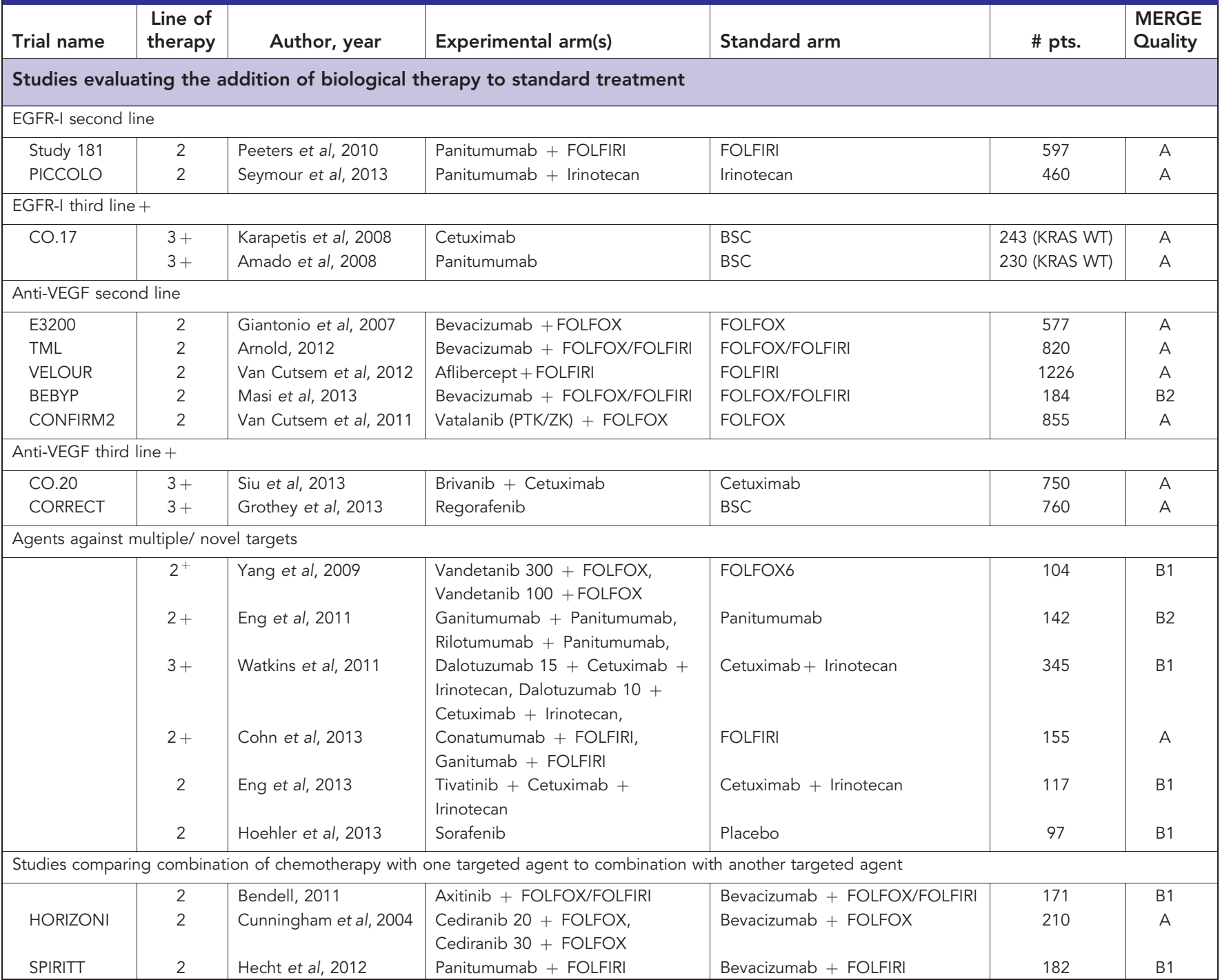

GROUP 1: THE EFFECT OF ANY BIOLOGIC AGENT ADDED TO STANDARD THERAPY

Overall survival. Fifteen studies, involving 17 comparisons, reported OS HRs. Using fixed-effects meta-analysis the OS HR was 0.87 (95\% CI 0.82-0.91, $P<0.00001$, Figure 1$)$. As expected, there was significant heterogeneity given variation in type of agent and clinical settings (second and third-line therapy). Randomeffects analysis was, therefore, performed confirming OS benefit with HR 0.88 (95\% CI 0.81-0.97, $P=0.008$ ).

Progression-free survival. Seventeen studies involving 21 comparisons demonstrated PFS benefit for use of biological agents with fixed-effects HR 0.71 (95\% CI 0.67-0.74, $P<0.0001$, Figure 2) and random-effects analysis (given heterogeneity) HR 0.75 (95\% CI $0.67-0.85, P<0.0001)$.

Overall response rate. Fourteen studies involving 17 comparisons allowed fixed-effects meta-analysis, which demonstrated pooled RR benefit of $+8.6 \%$ (OR 2.38; 95\% CI 2.03-2.78, $P<0.00001$, Figure 3). Despite significant heterogeneity, benefit was preserved on random-effects modelling with OR 2.04 (95\% CI $1.48-2.86$, $P<0.0001)$.
Toxicity. Fifteen studies involving 19 comparisons were analysed and demonstrated, significantly, increased risk of Grade 3/4 toxicity with OR 2.34 (95\% CI 2.12-2.59, $P<0.00001$, Figure 4) on fixed-effects modelling and OR 2.14 (95\% CI 1.70-2.69, $P<0.00001)$ on random-effects modelling.

Fourteen studies involving 18 comparisons reported Grade 5 (fatal) toxicity. The addition of a targeted agent did not significantly increase the risk of Grade 5 toxicity with pooled rate $+0.6 \%$ and OR 1.14 (95\% CI $0.72-1.81, P=0.58)$, with minimal heterogeneity.

\section{SUBGROUP ANALYSIS BY TARGETED THERAPY CLASS}

EGFR inhibitors. Considered as a group, the use of EGFR-I for KRAS WT patients in any setting was associated with a benefit to OS with HR 0.87 (95\% CI $0.77-0.97, P=0.01)$, PFS with HR 0.62 (95\% CI $0.55-0.70, P<0.00001)$ and ORR with pooled benefit $+21.6 \%$ and OR 5.30 (95\% CI 3.85-7.30, $P<0.00001$ ). Significant heterogeneity was present, and on random-effects modelling, OS benefit was no longer apparent with HR 0.84 (95\% CI 0.66-1.06, $P=0.15)$ but PFS benefit was preserved with HR 0.57 (95\% CI $0.42-0.79, P=0.0007)$. 


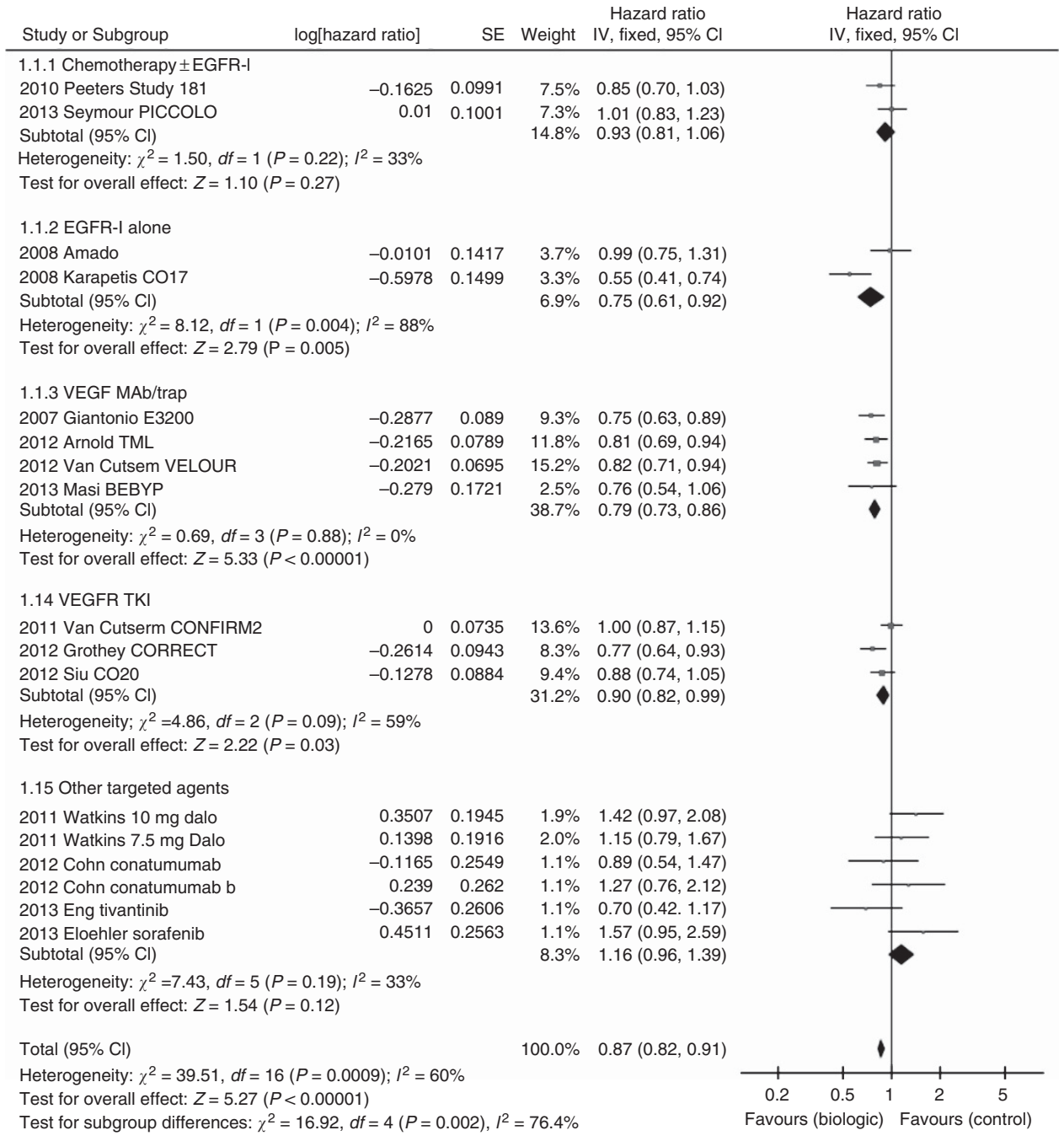

Figure 1. Forest plot for OS.

EGFR-I in second-line setting. Two trials - Study 181 (Peeters et al, 2010) and PICCOLO (Seymour et al, 2013) - investigated EGFR-I in the second-line setting, both in combination with (irinotecan-based) chemotherapy. Meta-analysis in 1057 KRAS WT patients demonstrated no improvement in OS with HR 0.93 (95\% CI 0.81-1.06, $P=0.27$ ), however, significant improvement in PFS with HR 0.76 (95\% CI 0.65-0.87, $P=0.0002)$ and ORR with pooled RR $+24.0 \%$ and OR 4.44 (95\% CI 3.20-6.18, $P<0.00001)$. No significant heterogeneity was present.

EGFR-I in third-line setting and beyond. Two trials (CO.17 (Karapetis et al, 2008) and Amado 2008 (Amado et al, 2008)) were identified involving 473 KRAS WT patients; both used EGFR-I as monotherapy. Benefit was demonstrated for OS with HR 0.75 (95\% CI $0.61-0.92, P=0.005$ ), PFS with HR 0.42 (95\% CI $0.35-0.52$, $P<0.0001)$, and ORR with pooled RR $+16 \%$ and OR $42.29(95 \%$ CI 5.76-310.58, $P=0.0002$ ).

Significant heterogeneity was noted for OS, likely attributable to crossover in the Amado study. Using a random-effects model, the OS benefit was no longer significant with HR 0.74 (95\% CI $0.42-$ $1.32, P=0.30)$.

Anti-angiogenic agents. The use of any anti-angiogenic agent in any setting was associated with OS benefit with HR 0.84 (95\% CI $0.79-0.89, P<0.00001$ ), PFS benefit with HR 0.68 (95\% CI $0.64-$ $0.72, P<0.00001)$ and ORR with pooled $\mathrm{RR}+4.8 \%$ and OR 2.01 (95\% CI 1.62-2.49, $P<0.00001)$. There was considerable statistical heterogeneity for PFS, attributable to differences in class of drug, line of therapy and prior anti-angiogenic treatment. Randomeffects meta-analysis showed preservation of benefit, with PFS HR of 0.67 (95\% CI $0.59-0.77, P<0.00001)$.

Anti-angiogenic agents in second-line setting. Five trials involving 3662 patients E3200 (Giantonio et al, 2007), TML (Bennouna et al, 2013), VELOUR (Van Cutsem et al, 2012), BEBYP (Masi et al, 2013), CONFIRM2 (Van Cutsem et al, 2011)) were identified investigating the addition of bevacizumab, aflibercept or vatalanib in the second-line setting. Fixed-effects meta-analysis demonstrated benefit for OS with HR 0.84 (95\% CI 0.78-0.91, $P<0.00001$ ), PFS with HR 0.72 (95\% CI $0.67-0.77, P<0.00001$ ), and ORR with pooled RR $+7.2 \%$ and OR 2.00 (95\% CI 1.57-2.54, $P<0.00001)$. Heterogeneity in ORR was noted but benefit was preserved using random-effects modelling with OR 1.89 (95\% CI $1.28-2.80, \quad P=0.001)$. Sensitivity analysis with exclusion of BEBYP(Masi et al, 2013) study (judged as lesser quality owing to poor accrual) did not alter results, retaining benefit to OS (HR 0.85), PFS (HR 0.72) and ORR (OR 2.11).

Anti-angiogenic agents in third-line setting and beyond. Two trials - CORRECT (Grothey et al, 2013) and CO.20 (Siu et al, 2013) - were analysed investigating regorafenib and brivanib, respectively, in a total of 1510 patients. Benefit was shown for OS with HR 0.83 (95\% CI $0.73-0.94, P=0.003)$, PFS with HR 0.60 (95\% CI $0.54-0.67, P<0.00001)$ and ORR with pooled RR $+0.9 \%$ 


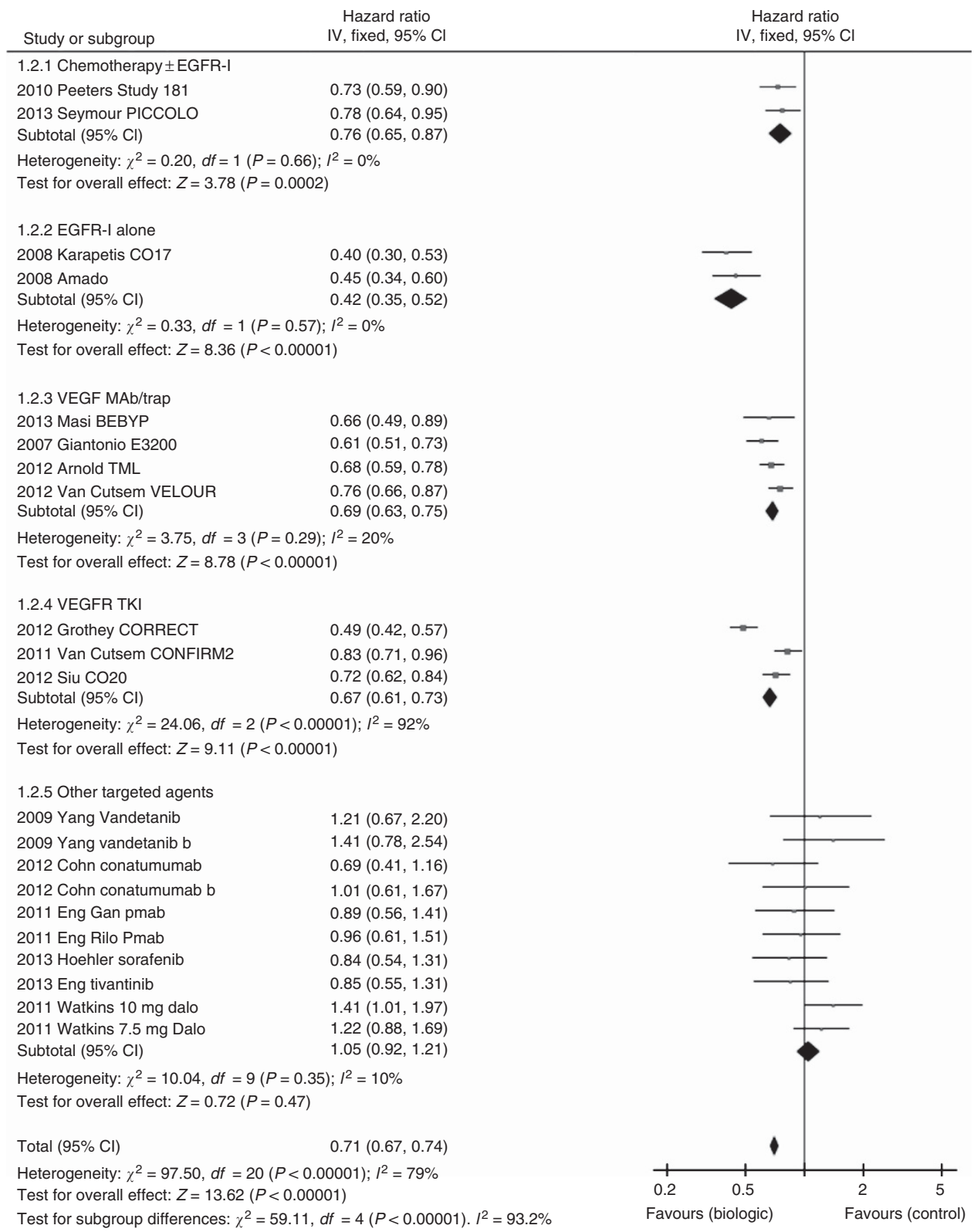

Figure 2. Forest plot for PFS.

and OR 2.05 (95\% CI 1.27-3.30, $P=0.003$ ). Given considerable heterogeneity in PFS, random-effects modelling was performed, which preserved PFS benefit (HR 0.59, 95\% CI 0.41-0.87, $P=0.007)$. The heterogeneity was thought to be predominantly due to the different study settings and populations. The CORRECT study examined the effect of regorafenib in chemotherapyrefractory patients, of which $48 \%$ had received four or more prior lines of therapy but all were ECOG 0-1. The CO.20 trial investigated KRAS WT patients with both arms receiving cetuximab, of whom $91 \%$ had received four or more prior lines of therapy but that also allowed enrolment of ECOG two patients.

Other targeted agents. Six trials involving 960 patients investigated the addition of targeted agents not primarily directed against EGFR or VEGF/VEFGR - namely, conatumumab, ganitumab, dalotuzumab, rilotumumab, tivantinib, sorafenib and vandetanib. Given the varied modes of action of the above agents, metaanalysis was not performed.

Sensitivity analysis. Remodelling of analysis of overall effect to exclude the six trials of 'other targeted agents', as they are not currently used in clinical practice, preserved benefit in OS with HR 0.84 (95\% CI $0.80-0.89, P<0.00001)$, PFS with HR 0.67 (95\% CI $0.63-0.70, P<0.00001)$ and ORR with OR 2.79 (95\% CI 2.34-3.33, $P<0.00001)$.

Given the high degree of crossover in the Amado study, the effect of its exclusion from analysis of all EGFR-I (1.1) was investigated. A significant benefit to OS was maintained on fixedeffects modelling (HR 0.84, 95\% CI 0.74-0.95) but was again absent on random-effects modelling (HR 0.79, 95\% CI 0.58-1.08).

Similarly, analysis to include data from the EPIC trial (entire cohort) did not change results for second-line EGFR-I, with OS HR 0.95 (95\% CI 0.86-1.05), PFS HR 0.72 (95\% CI 0.65-0.78), ORR OR 4.46 (95\% CI 3.43-5.81).

\section{GROUP 2: COMPARING ONE BIOLOGIC THERAPY WITH} ANOTHER

There were only three eligible trials, with five comparisons, in a total of 551 patients, where one biologic therapy (axitinib, 


\begin{tabular}{|c|c|c|c|c|c|c|c|c|c|}
\hline \multirow[b]{2}{*}{ Study or subgroup } & \multicolumn{2}{|c|}{ Biologic } & \multicolumn{2}{|c|}{ Control } & \multirow{2}{*}{$\begin{array}{c}\text { Odds ratio } \\
\mathrm{M}-\mathrm{H} \text {, fixed, } 95 \% \mathrm{Cl}\end{array}$} & \multirow{2}{*}{\multicolumn{3}{|c|}{$\begin{array}{c}\text { Odds ratio } \\
\mathrm{M}-\mathrm{H} \text {, fixed, } 95 \% \mathrm{Cl}\end{array}$}} & \\
\hline & Events & Total & Events & Total & & & & & \\
\hline \multicolumn{10}{|l|}{ 1.3.1 Chemotherapy \pm EGFR-I } \\
\hline 2010 Peeters Study 181 & 106 & 303 & 29 & 294 & $4.92(3.13,7.71)$ & & & - & \\
\hline 2013 Seymour PICCOLO & 79 & 230 & 27 & 230 & $3.93(2.42,6.39)$ & & & $\rightarrow$ & \\
\hline Subtotal $(95 \% \mathrm{Cl})$ & & 533 & & 524 & $4.44(3.20,6.18)$ & & & $\boldsymbol{\varphi}$ & \\
\hline Total events & 185 & & 56 & & & & & & \\
\hline \multicolumn{10}{|c|}{ Heterogeneity: $\chi^{2}=0.44, d f=1(P=0.51) ; I^{2}=0 \%$} \\
\hline \multicolumn{10}{|c|}{ Test for overall effect: $Z=8.87(P<0.00001)$} \\
\hline \multicolumn{10}{|l|}{ 1.3.2 EGFR-I alone } \\
\hline 2008 Amado & 21 & 108 & 0 & 103 & $50.86(3.04,851.84)$ & & & & \\
\hline 2008 Karapetis $\mathrm{CO} 17$ & 15 & 117 & 0 & 113 & $34.33(2.03,580.98)$ & & & & \\
\hline Subtotal $(95 \% \mathrm{Cl})$ & & 225 & & 216 & $42.29(5.76,310.58)$ & & & & \\
\hline Total events & 36 & & 0 & & & & & & \\
\hline \multicolumn{10}{|c|}{ Heterogeneity: $\chi^{2}=0.04, d f=1(P=0.85) ; l^{2}=0 \%$} \\
\hline \multicolumn{10}{|c|}{ Test for overall effect: $Z=3.68(P<0.00002)$} \\
\hline \multicolumn{10}{|l|}{ 1.3.3 VEGF MAb/trap } \\
\hline 2007 Giantonio E3200 & 65 & 286 & 25 & 291 & $3.13(1.91,5.13)$ & & & $\rightarrow$ & \\
\hline 2012 Arnold TML & 22 & 404 & 16 & 406 & $1.40(0.73,2.71)$ & & & $\leftarrow$ & \\
\hline 2012 Van Cutsem VELOUR & 105 & 531 & 59 & 530 & $1.97(1.39,2.78)$ & & & \# & \\
\hline 2013 Masi BEBYP & 19 & 92 & 17 & 92 & $1.15[0.55,2.38)$ & & & - & \\
\hline Subtotal $(95 \% \mathrm{Cl})$ & & 1313 & & 1319 & $2.00(1.57,2.54)$ & & & $\bullet$ & \\
\hline Total events & 211 & & 117 & & & & & & \\
\hline \multicolumn{10}{|c|}{ Heterogeneity: $\chi^{2}=6.49, d f=3(P=0.09) ; l^{2}=54 \%$} \\
\hline \multicolumn{10}{|c|}{ Test for overall effect: $Z=5.58(P<0.00001)$} \\
\hline \multicolumn{10}{|l|}{ 1.3.4 VEGFR TKI } \\
\hline 2012 Grothey CORRECT & 5 & 500 & 1 & 255 & $2.57(0.30,22.08)$ & & & & \\
\hline 2012 Siu CO20 & 51 & 376 & 27 & 374 & $2.02(1.24,3.29)$ & & & $\rightarrow$ & \\
\hline Subtotal $(95 \% \mathrm{Cl})$ & & 876 & & 629 & $2.05(1.27,3.30)$ & & & $\bullet$ & \\
\hline Total events & 56 & & 28 & & & & & & \\
\hline \multicolumn{10}{|c|}{ Heterogeneity: $\chi^{2}=0.05, d f=1(P=0.83) ; l^{2}=0 \%$} \\
\hline \multicolumn{10}{|c|}{ Test for overall effect: $Z=2.94(P=0.003)$} \\
\hline \multicolumn{10}{|l|}{ 1.3.5 Other targeted agents } \\
\hline 2011 Eng Gan pmab & 10 & 46 & 10 & 48 & $1.06(0.39,2.83)$ & & & - & \\
\hline 2011 Eng Rilo Pmab & 15 & 48 & 10 & 48 & $1.73(0.68,4.36)$ & & & . & \\
\hline 2011 Watkins $10 \mathrm{mg}$ dalo & 25 & 116 & 14 & 55 & $0.80(0.38,1.70)$ & & & - & \\
\hline 2011 Watkins $7.5 \mathrm{mg}$ Dalo & 28 & 117 & 14 & 55 & $0.92(0.44,1.93)$ & & & - & \\
\hline 2012 Cohn conatumumab & 7 & 51 & 1 & 26 & $3.98(0.46,34.21)$ & & & & \\
\hline 2012 Cohn conatumumab b & 4 & 51 & 1 & 26 & $2.13(0.23,20.07)$ & & & & \\
\hline 2013 Eng tivantinib & 27 & 60 & 19 & 57 & $1.64(0.77,3.46)$ & & & $\rightarrow$ & \\
\hline 2013 Eloehler sorafenib & 0 & 0 & 0 & 0 & Not estimable & & & & \\
\hline Subtotal $(95 \% \mathrm{Cl})$ & & 489 & & 315 & $1.23(0.86,1.74)$ & & & 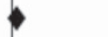 & \\
\hline Total events & 116 & & 69 & & & & & & \\
\hline \multicolumn{10}{|c|}{ Heterogeneity: $\chi^{2}=4.35, d f=6(P=0.63) ; I^{2}=0 \%$} \\
\hline Test for overall effect: $Z=1.15$ & $\mathrm{P}=0.25)$ & & & & & & & & \\
\hline Total $(95 \% \mathrm{Cl})$ & & 3463 & & 3003 & $2.38(2.03,2.78)$ & & & 1 & \\
\hline Total events & 604 & & 270 & & & & & & \\
\hline Heterogeneity: $\chi^{2}=49.70, d f=$ & $6(P<0.0$ & 001); $I^{2}$ & $2=68 \%$ & & & $\longmapsto$ & 1 & +1 & \\
\hline Test for overall effect: $Z=10.8$ & $(P<0.000$ & $001)$ & & & & 0.001 & 0.1 & 10 & 1000 \\
\hline Test for subgroup differences: & $=37.23$ & $d f=4(F$ & $P<0.000$ & $01), l^{2}$ & $9.3 \%$ & Favo & (control) & Favours & ologic) \\
\hline
\end{tabular}

Figure 3. Forest plot for ORR.

cediranib and panitumumab) was compared with different biologic (bevacizumab) added to the same standard treatment, so that the trials were appropriate for comparison. There was no significant overall difference in OS for any of the experimental biological agents with HR 1.07 (95\% CI $0.88-1.29, P=0.51$, Figure 5), PFS with HR 1.14 (95\% CI $0.93-1.40, P=0.20$, Supplementary Figure 6) and ORR with OR 0.69 (95\% CI 0.46$1.04, P=0.07$ )

No significant difference in the incidence of overall Grade 3/4 toxicity was present with OR 0.70 (95\% CI 0.40-1.20), $P=0.19$ (Figure 6).

Quality of life. Only 5 of the 20 studies reported QoL data (Table 2). The two studies of EGFR-I reported significant QoL improvement: CO.17 with cetuximab monotherapy vs BSC and the PICCOLO study in second-line treatment examining cetuximab with chemotherapy. By contrast, significant deterioration in QoL was recorded with the addition of brivanib to cetuximab in the CO.20 study. Regorafenib and cediranib did not alter QoL.

\section{DISCUSSION}

Despite the sometimes nihilistic view of lack of progress in the management of patients with mCRC, when considered as a therapeutic class, the addition of either EGFR or angiogenesis inhibitors to standard therapy for mCRC beyond the first-line setting has impacted positively on overall survival as well as progression-free survival. As a group, these agents also improved overall response rate and did not diminish overall QOL. As expected, the rate of any Grade 3/4 toxicity was increased, but there was no increase in treatment-related mortality. The size of benefit is analysed in detail in this systematic review, to inform discussion regarding best placement of each biological agent in the treatment paradigm, as the optimal sequencing of these drugs has not been clearly defined. Cost-effectiveness, not analysed here, is also important.

There are several strengths to this study. By identifying all relevant trials, it rigorously shows that the addition of biological 


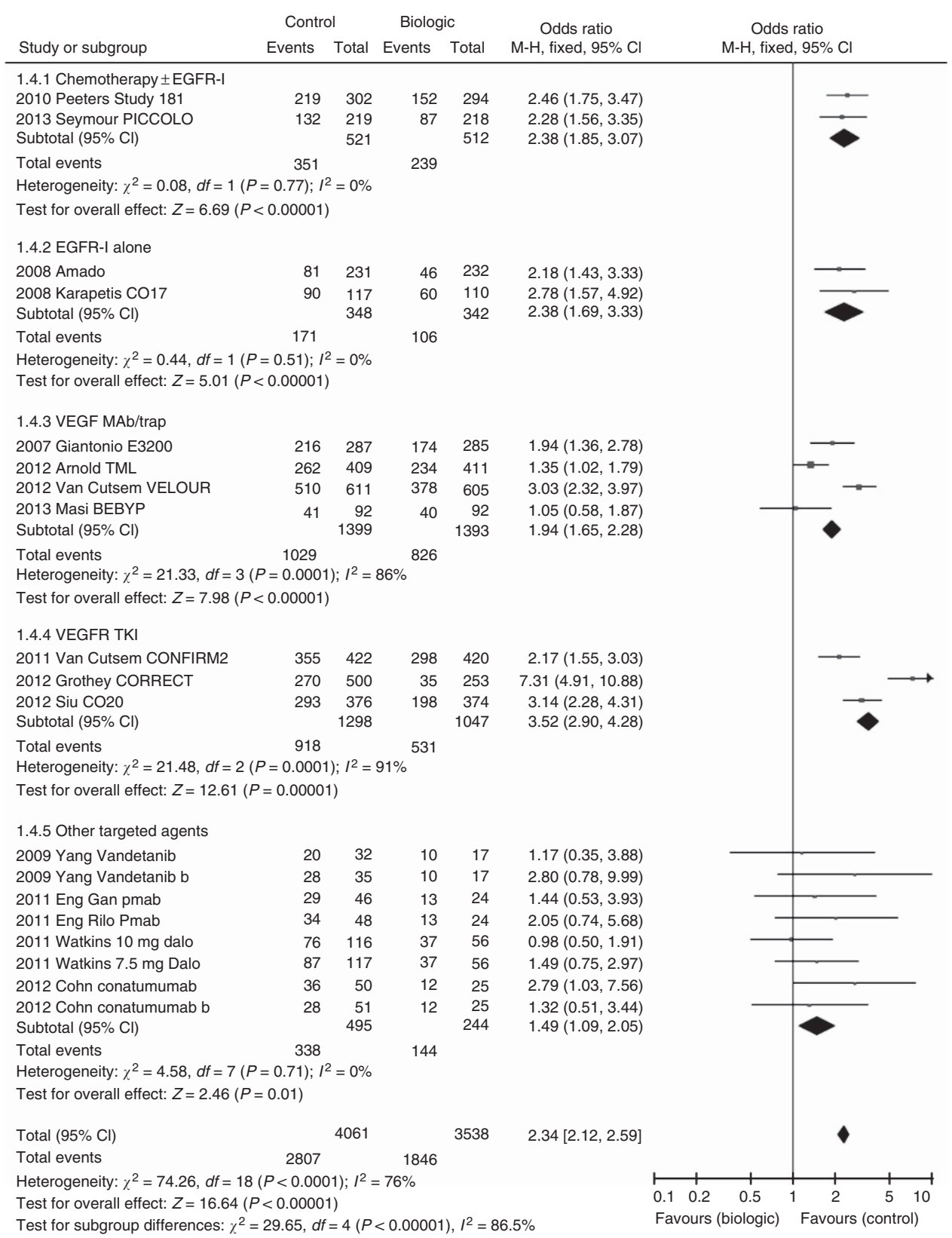

Figure 4. Forest plot for Grade 3/4 toxicity.

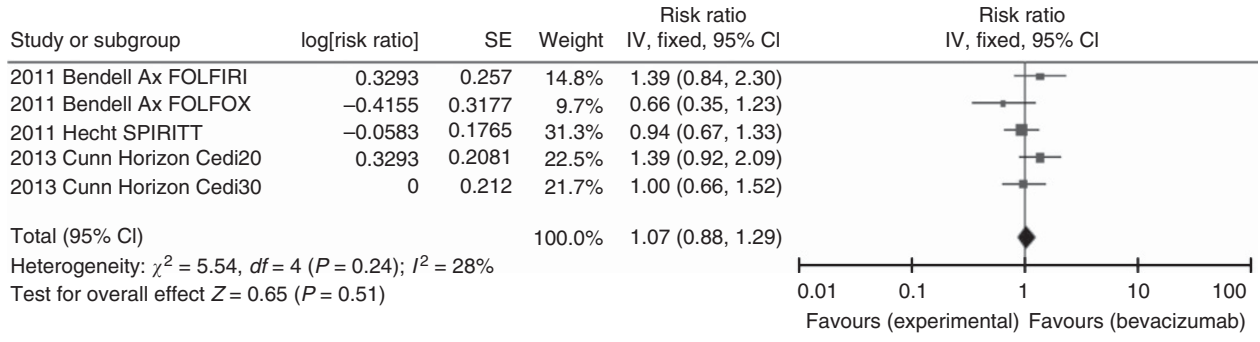

Figure 5. Forest plot for OS-chemotherapy + targeted agent 1 vs chemotherapy + bevacizumab.

therapy improves outcomes. The large number of trials and patients lent itself to a meta-analytic approach.

There are also limitations to the current study. The metaanalysis of anti-angiogenic agents as a group provides a broad answer to the questions posed as it pools both bevacizumab and aflibercept data together in analysis, despite slightly differing modes of action. The number of trials in some subgroups is small.
This makes random-effects modelling less certain. It also highlights the difficulty in performing subsequent trials with new agents once proof of efficacy is established. The use of individual patient data would have been ideal, and work is underway to obtain this information to enable further analysis.

With respect to agents targeting EGFR, the monoclonal antibodies cetuximab and panitumumab overall added OS and 


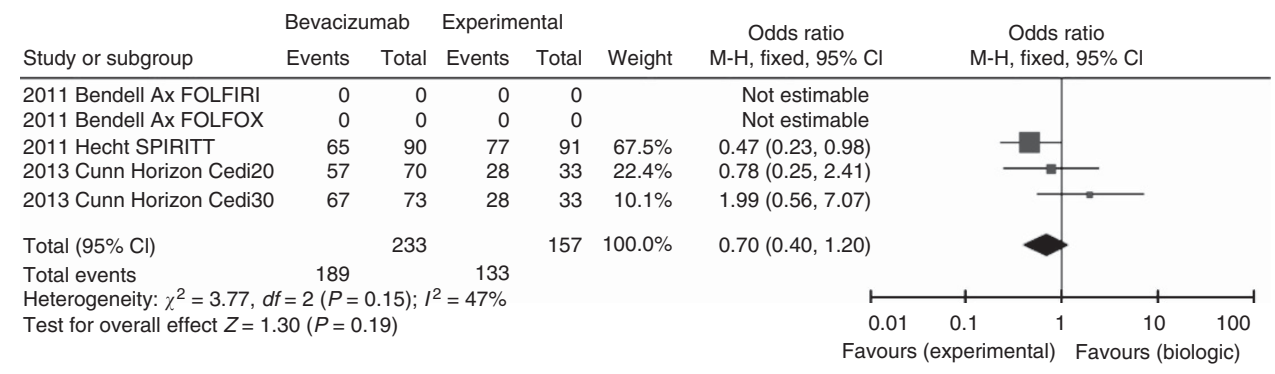

Figure 6. Forest plot for Grade 3/4 toxicity-chemotherapy + targeted agent 1 vs chemotherapy + bevacizumab.

\begin{tabular}{|c|c|c|c|c|c|c|c|}
\hline $\begin{array}{l}\text { Study } \\
\text { title }\end{array}$ & Treatment & Control & $N$ & QoL instrument & QoL effect & $P$-value & Details \\
\hline PICCOLO & $\begin{array}{l}\text { Irinotecan }+ \\
\text { Panitumumab (IrPan) }\end{array}$ & Irinotecan & 597 & $\begin{array}{l}\text { EORTC QLQ-C30, } \\
\text { EQ-5D, } \\
\text { dermatology life quality } \\
\text { index }\end{array}$ & $\begin{array}{l}\text { Significantly } \\
\text { better } \\
\text { No data }\end{array}$ & $\begin{array}{l}0.032 \\
\text { N/A }\end{array}$ & $\begin{array}{l}\text { QLQ-C30 global scores } \\
\text { favoured IrPan group ( } 56.4 \\
\text { vs. 49.5), but QoL symptom } \\
\text { scores worse with IrPan }\end{array}$ \\
\hline CO.17 & Cetuximab & BSC & 243 (KRAS WT) & EORTC QLQ-C30 & $\begin{array}{l}\text { Significantly } \\
\text { better }\end{array}$ & 0.0002 & $\begin{array}{l}\text { Mean diff at } 8 \text { wk } 10.9(95 \% \\
\mathrm{Cl} 4.2-17.6, P=0.0002), 16 \\
\text { wk } 17.9(95 \% \mathrm{Cl} 7.6-28.2 \\
P<0.0001)\end{array}$ \\
\hline CO.20 & Brivanib + Cetuximab & Cetuximab & 750 & EORTC QLQ-C30 & $\begin{array}{l}\text { Significantly } \\
\text { worse }\end{array}$ & 0.02 & $\begin{array}{l}\text { Global and physical QoL } \\
\text { scores deteriorated } \\
\text { significantly faster in brivanib } \\
\text { arm compared with placebo }\end{array}$ \\
\hline CORRECT & Regorafenib & $\mathrm{BSC}$ & 760 & $\begin{array}{l}\text { EORTC QLQ-C30, EQ- } \\
\text { 5D index, EQ-5D VAS }\end{array}$ & $\begin{array}{l}\text { Not } \\
\text { significantly } \\
\text { different }\end{array}$ & N/A & \\
\hline Cediranib & $\begin{array}{l}\text { Cediranib } 20+ \\
\text { FOLFOX, Cediranib } \\
30+\text { FOLFOX }\end{array}$ & $\begin{array}{l}\text { Bevacizumab } \\
+ \text { FOLFOX }\end{array}$ & 210 & $\begin{array}{l}\text { FACT-C symptom index } \\
\text { (FCSI) subscale; trial } \\
\text { outcome index (TOI), } \\
\text { total FACT score }\end{array}$ & $\begin{array}{l}\text { Not } \\
\text { significantly } \\
\text { different }\end{array}$ & $\begin{array}{l}0.15 \text { (FCSI } \\
\text { Cedi 20), } \\
0.22 \text { (FCSI } \\
\text { Cedi 30) }\end{array}$ & $\begin{array}{l}\text { Time to worsening of TOI, } \\
\text { Total FACT score also } \\
\text { non-significant }\end{array}$ \\
\hline
\end{tabular}

PFS benefit to standard therapy. However, no OS advantage was seen when used in the second-line setting (in combination with chemotherapy), although there was benefit for PFS and ORR. The lack of OS benefit is most likely attributable to the crossover to later receive anti-EGFR therapy, which occurred in both trials. Subsequent EGFR-I use, allowed by protocol, was reported in 31\% of patients in the FOLFIRI-only arm of Study, 181 compared with $10 \%$ in the FOLFIRI-panitumumab arm, although only in $6 \%$ of the chemotherapy alone arm in the PICCOLO study, compared with $<0.5 \%$ in the irinotecan-panitumumab arm.

The impact of crossover is strikingly demonstrated in the thirdline setting. In the CO.17 study, which demonstrated significant OS benefit, crossover was not allowed from BSC to cetuximab, with only 13/285 patients later receiving EGFR-I. By contrast, in the Amado trial, which allowed crossover, this occurred in 90/119 KRAS WT patients resulting in no OS benefit being demonstrated. Even with the exclusion of the Amado study from analysis, however, no OS benefit was demonstrated on random-effects modelling.

Another explanation for the lack of OS benefit of the EGFR-I in the second-line setting is the issue of patient selection. New data strongly support tumour testing for additional RAS mutations, which have also been shown to be robust negative predictive factors for EGFR-I response. Extended KRAS testing beyond the traditional examination of exon 2 to include mutational hotspots in exons 3 and 4 , as well as the NRAS gene (exons 2, 3 and 4) and (in some series) PI3KCA exon 20, has been established from the analysis of several first-line EGFR-I trials:
PRIME (Douillard et al, 2013) and FIRE-3 (Stintzing et al, 2012). Extended mutation analysis in the EFGR-I studies in second-line therapy may alter the OS data in the 'pure' WT subgroups and, these data are eagerly awaited. As both studies in the second-line EGFR-I analysis used irinotecan backbones, concerns regarding possible negative interactions between oxaliplatin and EGFR-I are not relevant.

Current opinion is divided on best placement of EGFR-I use in the treatment pathway of mCRC. In first-line therapy of patients with KRAS WT mCRC, the use of an EGFR-I with chemotherapy has to be considered against an anti-VEGF agent. There are many countries where first-line EGFR-I is not freely available; additional concerns were recently raised by the New EPOC study (Primrose et al, 2013) for a possible detrimental effect, at least in the trial population of resectable liver metastases. In contrast, data from the FIRE-3 study (Stintzing et al, 2012), directly comparing cetuximab with bevacizumab in the first-line setting and available as abstract only, showed a significant OS advantage for first- line cetuximab. However this was a secondary end point and there was no difference in the primary end point of RR nor in PFS or downstaging to resection. The late separation of survival curves does point to possible ongoing influence of first-line biologic choice, which appears more exaggerated with selection by extended RAS testing (Stintzing et al, 2012).

Regarding anti-angiogenesis agents as a group, their addition to chemotherapy backbones was associated with improved OS, PFS and ORR. This benefit was present whether they were used in second-line settings or third-line settings and beyond. 
The VEGF antibody bevacizumab and the VEGFtrap aflibercept had remarkably similar efficacy (1.4 months median OS benefit), despite different patient populations (30\% prior exposure to bevacizumab in VELOUR study; 100\% in TML study). This may be due to continuous VEGF inhibition, which may be important throughout the refractory setting, as a proportion of patients in the CORRECT trial population had been bevacizumab pre-treated. Vatalanib failed to enter clinical practice because it did not meet its primary end point of OS despite demonstration of PFS benefit. The lack of robust predictive biomarkers in anti-angiogenic therapy remains a major challenge.

Anti-angiogenesis trials in the third-line and beyond setting investigated the VEGFR TKIs regorafenib and brivanib. Considered together, benefit was shown for OS, PFS and ORR. Only regorafenib has moved into standard of care; however, predictive markers remain elusive. Brivanib has not entered routine clinical practice, despite significant benefit in ECOG 0-1 patients (compared with ECOG 2) in the CO.20 trial, primarily due to adverse effects on QoL.

Many studies of targeted agents did not include formal QoL measurements. This is a lost opportunity. Despite some of these trials achieving the gold standard of OS improvement, the drugs remain non-curative and it is always necessary to consider the impact of treatment-related side effects on global and specific functioning. The CO.20 trial illustrates the importance of measuring QoL, as brivanib may otherwise have been taken forward in the good performance status group.

\section{CONCLUSIONS}

This systematic review has provided evidence for a class effect with the addition of targeted therapies, when considered together, improving OS, PFS and ORR for patients with mCRC. When analysed separately by mechanism of action and by line of therapy, results demonstrate that progress has been made in the extension of life of patients with mCRC.

\section{ACKNOWLEDGEMENTS}

We acknowledge the authors who provided additional data: B Giantonio, J Tabernero, C O'Callaghan and D Jonker. SanofiAventis provided financial support to WriteSource Medical Pty Ltd to undertake the literature search and paper retrieval. SanofiAventis had no role in data analysis/interpretation and were not involved in writing the manuscript.

\section{REFERENCES}

Amado RG, Wolf M, Peeters M, Van Cutsem E, Siena S, Freeman DJ, Juan T, Sikorski R, Suggs S, Radinsky R, Patterson SD, Chang DD (2008) Wild-type KRAS is required for panitumumab efficacy in patients with metastatic colorectal cancer. J Clin Oncol 26(10): 1626-1634.

Arnold D, Andre T, Bennouna J, Sastre J, Osterlund PJ, Greil R, Van Cutsem E, Von Moos R, Reyes-Rivera I, Bendahmane B, Kubicka S (2012) Bevacizumab (BEV) plus chemotherapy (CT) continued beyond first progression in patients with metastatic colorectal cancer (mCRC) previously treated with BEV plus CT: results of a randomized phase III intergroup study (TML study). J Clin Oncol 30(suppl): abstract CRA3503.

Bendell JC, Tournigand C, Bednarczyk M, Swieboda-Sadlej A, Chung I, Barone C, Tarazi JC, Rosbrook B, Ricart AD, Sobrero AF (2011) Axitinib or bevacizumab (bev) plus FOLFOX or FOLFIRI as second-line therapy in patients (pts) with metastatic colorectal cancer (mCRC). J Clin Oncol 29(suppl 4): abstract 478.

Bennouna J, Sastre J, Arnold D, Osterlund P, Greil R, Van Cutsem E, von Moos R, Vieitez JM, Bouche O, Borg C, Steffens CC, Alonso-Orduna V,
Schlichting C, Reyes-Rivera I, Bendahmane B, Andre T, Kubicka S. Investigators MLS (2013) Continuation of bevacizumab after first progression in metastatic colorectal cancer (ML18147): a randomised phase 3 trial. Lancet Oncol 14(1): 29-37.

Cohn AL, Tabernero J, Maurel J, Nowara E, Sastre J, Chuah BY, Kopp MV, Sakaeva DD, Mitchell EP, Dubey S, Suzuki S, Hei YJ, Galimi F, McCaffery I, Pan Y, Loberg R, Cottrell S, Choo SP (2013) A randomized, placebocontrolled phase 2 study of ganitumab or conatumumab in combination with FOLFIRI for second-line treatment of mutant KRAS metastatic colorectal cancer. Ann Oncol 24(7): 1777-1785.

Cunningham D, Humblet Y, Siena S, Khayat D, Bleiberg H, Santoro A, Bets D, Mueser M, Harstrick A, Verslype C, Chau I, Van Cutsem E (2004) Cetuximab monotherapy and cetuximab plus irinotecan in irinotecanrefractory metastatic colorectal cancer. N Engl J Med 351(4): 337-345.

Douillard JY, Oliner KS, Siena S, Tabernero J, Burkes R, Barugel M, Humblet Y, Bodoky G, Cunningham D, Jassem J, Rivera F, Kocakova I, Ruff P, Blasinska-Morawiec M, Smakal M, Canon JL, Rother M, Williams R, Rong A, Wiezorek J, Sidhu R, Patterson SD (2013) Panitumumab-FOLFOX4 treatment and RAS mutations in colorectal cancer. N Engl J Med 369(11): 1023-1034.

Eng C, Hart LL, Severtsev A, Gladkov O, Mueller L, Kopp MV, Vladimirov VI, Langdon RM, Kotiv B, Barni S, Hsu C, Bolotin E, Von Roemeling R, Schwartz BE, Bendell JC (2013) A randomized, placebo-controlled, phase I/II study of tivantinib (ARQ 197) in combination with cetuximab and irinotecan in patients (pts) with KRAS wild-type (WT) metastatic colorectal cancer (CRC) who had received previous front-line systemic therapy. ASCO Meeting Abstracts 31(suppl): 3508.

Eng C, Van Cutsem E, Nowara E, Swieboda-Sadlej A, Tebbutt NC, Mitchell EP, Davidenko I, Oliner K, Chen L, Huang J, McCaffery I, Loh E, Smethurst D, Tabernero J (2011) A randomized, phase Ib/II trial of rilotumumab (AMG 102; ril) or ganitumab (AMG 479; gan) with panitumumab (pmab) versus pmab alone in patients (pts) with wild-type (WT) KRAS metastatic colorectal cancer (mCRC): Primary and biomarker analyses. ASCO Meeting Abstracts 29(15_suppl): 3500.

Giantonio BJ, Catalano PJ, Meropol NJ, O’Dwyer PJ, Mitchell EP, Alberts SR, Schwartz MA, Benson 3rd AB. Eastern Cooperative Oncology Group Study E (2007) Bevacizumab in combination with oxaliplatin, fluorouracil, and leucovorin (FOLFOX4) for previously treated metastatic colorectal cancer: results from the Eastern Cooperative Oncology Group Study E3200. J Clin Oncol 25(12): 1539-1544.

Grothey A, Van Cutsem E, Sobrero A, Siena S, Falcone A, Ychou M, Humblet Y, Bouche O, Mineur L, Barone C, Adenis A, Tabernero J, Yoshino T, Lenz HJ, Goldberg RM, Sargent DJ, Cihon F, Cupit L, Wagner A, Laurent D, Group CS (2013) Regorafenib monotherapy for previously treated metastatic colorectal cancer (CORRECT): an international, multicentre, randomised, placebo-controlled, phase 3 trial. Lancet 381(9863): 303-312.

Hecht JR, Cohn AL, Dakhil SR, Saleh MN, Piperdi B, Cline-Burkhardt VJM, Tian Y, Go WY (2012) SPIRITT (study 20060141): A randomized phase II study of FOLFIRI with either panitumumab (pmab) or bevacizumab (bev) as second-line treatment (tx) in patients (pts) with wild-type (WT) KRAS metastatic colorectal cancer (mCRC). J Clin Oncol 30(Suppl 34): abstract 454 .

Hoehler TD, T, Schimanski C et al. (2013) Final results of the AIO 0307 study: A controlled, randomized, double-blind phase II study of FOLFOX6 or FOLFIRI combined with sorafenib $(\mathrm{S})$ versus placebo $(\mathrm{P})$ in second-line metastatic colorectal carcinoma (mCRC) treatment. ASCO Meeting Abstracts 31(Suppl): 3586.

Karapetis CS, Khambata-Ford S, Jonker DJ, O'Callaghan CJ, Tu D, Tebbutt NC, Simes RJ, Chalchal H, Shapiro JD, Robitaille S, Price TJ, Shepherd L, Au HJ, Langer C, Moore MJ, Zalcberg JR (2008) K-ras mutations and benefit from cetuximab in advanced colorectal cancer. N Engl J Med 359(17): 1757-1765.

Kontopantelis E, Springate DA, Reeves D (2013) A re-analysis of the Cochrane Library data: the dangers of unobserved heterogeneity in meta-analyses. PloS One 8(7): e69930.

Liddle J, Williamson M, Irwig L (1996) Method for evaluating research and guideline evidence. NSW Health Department: Sydney.

Masi G, Loupakis F, Salvatore L, Cremolini C, Fornaro L, Schirripa M, Granetto C, Miraglio E, Di Costanzo F, Antonuzzo L, Marcucci L, Barbara C, Boni C, Banzi M, Chiara S, Garbarino D, Valsuani C, Bonetti A, Boni L, Falcone A (2013) Second-line chemotherapy (CT) with or without bevacizumab (BV) in metastatic colorectal cancer (mCRC) patients (pts) who progressed to a first-line treatment containing $\mathrm{BV}$ : updated results of 
the phase III 'BEBYP' trial by the Gruppo Oncologico Nord Ovest (GONO). J Clin Oncol 31(suppl): abstract 3615.

Peeters M, Price TJ, Cervantes A, Sobrero AF, Ducreux M, Hotko Y, Andre T, Chan E, Lordick F, Punt CJ, Strickland AH, Wilson G, Ciuleanu TE, Roman L, Van Cutsem E, Tzekova V, Collins S, Oliner KS, Rong A, Gansert J (2010) Randomized phase III study of panitumumab with fluorouracil, leucovorin, and irinotecan (FOLFIRI) compared with FOLFIRI alone as second-line treatment in patients with metastatic colorectal cancer. J Clin Oncol 28(31): 4706-4713.

Primrose JN, Falk S, Finch-Jones M, Valle J, Sherlock D, Hornbuckle J, Gardner-Thrope J, Smith D, Imber C, Hickish T, Davidson B, Cunningham D, Poston GJ, Maughan T, Rees M, Stanton L, Little L, Bowers M, Wood W, Bridgewater JA (2013) A randomized clinical trial of chemotherapy compared to chemotherapy in combination with cetuximab in k-RAS wild-type patients with operable metastases from colorectal cancer: the new EPOC study. J Clin Oncol 31(suppl): abstract 3504 .

Seymour MT, Brown SR, Middleton G, Maughan T, Richman S, Gwyther S, Lowe C, Seligmann JF, Wadsley J, Maisey N, Chau I, Hill M, Dawson L, Falk S, O'Callaghan A, Benstead K, Chambers P, Oliver A, Marshall H, Napp V, Quirke P (2013) Panitumumab and irinotecan versus irinotecan alone for patients with KRAS wild-type, fluorouracil-resistant advanced colorectal cancer (PICCOLO): a prospectively stratified randomised trial. Lancet Oncol 14(8): 749-759.

Siu LL, Shapiro JD, Jonker DJ, Karapetis CS, Zalcberg JR, Simes J, Couture F, Moore MJ, Price TJ, Siddiqui J, Nott LM, Charpentier D, Liauw W, Sawyer MB, Jefford M, Magoski NM, Haydon A, Walters I, Ringash J, Tu D, O'Callaghan CJ (2013) Phase III randomized, placebo-controlled study of cetuximab plus brivanib alaninate versus cetuximab plus placebo in patients with metastatic, chemotherapy-refractory, wild-type K-RAS colorectal carcinoma: the NCIC Clinical Trials Group and AGITG CO.20 Trial. J Clin Oncol 31(19): 2477-2484.
Stintzing S, Fischer von Weikersthal L, Decker T, Vehling-Kaiser U, Jager E, Heintges T, Stoll C, Giessen C, Modest DP, Neumann J, Jung A, Kirchner T, Scheithauer W, Heinemann V (2012) FOLFIRI plus cetuximab versus FOLFIRI plus bevacizumab as first-line treatment for patients with metastatic colorectal cancer-subgroup analysis of patients with KRAS: mutated tumours in the randomised German AIO study KRK-0306. Ann Oncol 23(7): 1693-1699.

The Cochrane Collaboration Issues related to the unit of analysis - more than two treatment groups. Available at http://www.cochrane-net.org/ openlearning/html/modA2-5.htmVol. 2012.

Van Cutsem E, Bajetta E, Valle J, Kohne CH, Hecht JR, Moore M, Germond C, Berg W, Chen BL, Jalava T, Lebwohl D, Meinhardt G, Laurent D, Lin E (2011) Randomized, placebo-controlled, phase III study of oxaliplatin, fluorouracil, and leucovorin with or without PTK787/ZK 222584 in patients with previously treated metastatic colorectal adenocarcinoma. J Clin Oncol 29(15): 2004-2010.

Van Cutsem E, Tabernero J, Lakomy R, Prenen H, Prausova J, Macarulla T, Ruff P, van Hazel GA, Moiseyenko V, Ferry D, McKendrick J, Polikoff J, Tellier A, Castan R, Allegra C (2012) Addition of aflibercept to fluorouracil, leucovorin, and irinotecan improves survival in a phase III randomized trial in patients with metastatic colorectal cancer previously treated with an oxaliplatin-based regimen. J Clin Oncol 30(28): 3499-3506.

Watkins DJ, Tabernero J, Schmoll H, Trarbach T, Ramos FJ, Howe J, Brown HM, Clark J, Hsu K, Lu BD, Cunningham D (2011) A randomized phase II/III study of the anti-IGF-1R antibody MK-0646 (dalotuzumab) in combination with cetuximab $(\mathrm{Cx})$ and irinotecan (Ir) in the treatment of chemorefractory metastatic colorectal cancer (mCRC) with wild-type (wt) KRAS status. J Clin Oncol 29(Supp): Abstract 3501.

Yang TS, Oh DY, Guimbaud R, Szanto J, Salek T, Thurzo L, Vieitez JM, Pover GM, Kim TW (2009) Vandetanib plus mFOLFOX6 in patients with advanced colorectal cancer (CRC): a randomized, double-blind, placebo-controlled phase II study. ASCO Meeting Abstracts 27(15S): 4084.

Supplementary Information accompanies this paper on British Journal of Cancer website (http://www.nature.com/bjc) 